\title{
Towards Convolutional Neural Network Compression via $K$-Means Cluster
}

\author{
Guilin Chen ${ }^{1}$, Sheng $\mathrm{Ma}^{1}{ }^{+}$, and Yang Guo ${ }^{1}$ \\ ${ }^{1}$ College of computer, National University of Defense Technology, Changsha 410073
}

\begin{abstract}
With the rapid development of computer field, the computing power is no longer the bottleneck of the machine learning research. At present, deep learning technologies such as DNN (Deep Neural Networks) have been widely used in various fields such as speech recognition, smart driving, image recognition and so on. However, as the structure of DNN becomes more complex and the requirements of accuracy become higher, the network size is getting larger. The storage costs of such networks are high, which prohibits their usage in resource-constrained devices (embedded devices or mobile devices). In this paper, we propose a new compression method, compressing the DNN models without losing the accuracy by clustering the trained weights. Specifically, in our work, K-means is used to cluster the weights of fully connected layers. Then, we encode the clustered value as an index label. When we store the network model, we only store the index labels of weights. Compared with the original 32-bits weight, the index label is usually only three or four bits (based on the number of clusters), so as to achieve the purpose of compressing. Our compressing method achieves $11.4 \mathrm{x}$ compression rate on AlexNet network.
\end{abstract}

Keywords: neural network, compression, cluster, K-means.

\section{Introduction}

With the improvement of computing power, machine learning research began to pick up. The artificial neural network, as the representative of the deep learning technology, has developed rapidly. It has been widely applied to many applications of artificial intelligence, including image recognition [1], natural language processing [2] and robotics. Previously, the implementation platform of neural network is mainly a general-purpose processor, such as the graphics processing unit (GPU). However, due to the high computation demands of DNNs and the need for higher energy efficiency, researchers have begun to investigate specialized accelerators to speed up the processing of neural networks.

Steve Kecler, chief scientist of the NVIDIA, has once pointed out that the power consumption of transporting a 64-bit data for a distance of $20 \mathrm{~mm}$ is several times of 64-bit floating multiplication at $40 \mathrm{~nm}$ technology node [3]. Therefore, in order to reduce off-chip memory access and increase accelerator performance, we hope to store all the trained weight parameters on the accelerator chip in the design of accelerators. But it will take up a significant portion of chip area for weight storage, which compresses the processing module area. It is also unrealistic to store all the weights on-chip for large networks (such as AlexNet [4]). So, we consider compressing the weight file. By analyzing five representative neural networks (see Table I), we find that the parameters of fully connected layer are larger than the parameters of convolutional layer. Based on the above reasons, we propose a method of clustering compression to compress the fully connected layer of pre-trained neural networks. On the premise of maintaining the accuracy of the prediction, we want to make the compression rate is maximized as far as possible. The concrete idea of our method is to cluster the parameters of fully connected layer through $K$-means clustering algorithm. Each weight value has a corresponding clustering value. The clustering index value of each

+ Corresponding author. Tel.:13135213261

E-mail address: masheng@nudt.edu.cn 
weight and the clustered value of the full connection layer are stored on the chip. By storing the index, which is usually only three or four bits (based on the number of clusters) instead of the original 32-bits weight, we can achieve the purpose of compression. Compared with the quantification method proposed by Han Song [5], we cluster the pre-trained weight without retraining. Because there is no retraining, the accuracy of our compressed network may lose slightly. But in Minerva [6], we know that every network has a confidence interval of accuracy. If our compression method has an accuracy degradation below the acceptable range of confidence interval, its effect is indistinguishable from noise.

TABLE I: (C: CONVOLUTIONAL LAYER, F: FULLy CONNECTED LAYER)

\begin{tabular}{|c|c|c|c|}
\hline & Weights & C-weights-Rate & F-weights-Rate \\
\hline LeNet-300-100 & $1070 \mathrm{~KB}$ & - & $100 \%$ \\
LeNet-5 & $240 \mathrm{~KB}$ & $4.17 \%$ & $95.83 \%$ \\
Cifar10_quick & $600 \mathrm{~KB}$ & $56.92 \%$ & $43.08 \%$ \\
AlexNet & $240 \mathrm{MB}$ & $6.01 \%$ & $93.99 \%$ \\
VGG-16 & $528 \mathrm{MB}$ & $10.64 \%$ & $89.36 \%$ \\
\hline
\end{tabular}

The rest of this paper is structured as follows, Section 2 reviews some existing works for neural network compression. Section 3 puts forward the clustering compression algorithm and its theoretical basis. Section 4 introduces the experimental method, Section 5 gives the experimental results and analysis, and Section 6 concludes.

\section{Previous Neural Network Compression Work}

There are many schemes for neural network compression, and three different compression methods are introduced in [7] [8] and [9] respectively. Data-free pruning [7] reduces the weight parameter by 1.5 times, but the accuracy is greatly reduced. It mainly adopts the method of pruning similar neurons. If a similar weight pair can be found in the neural network, one of them is deleted, as shown in Figure 1. Unlike previous pruning operations, previous pruning usually removes a single weight, whereas Data-free pruning removes the entire neuron directly. It is noteworthy that the method is based on a trained model that does not require access to training data for retraining.

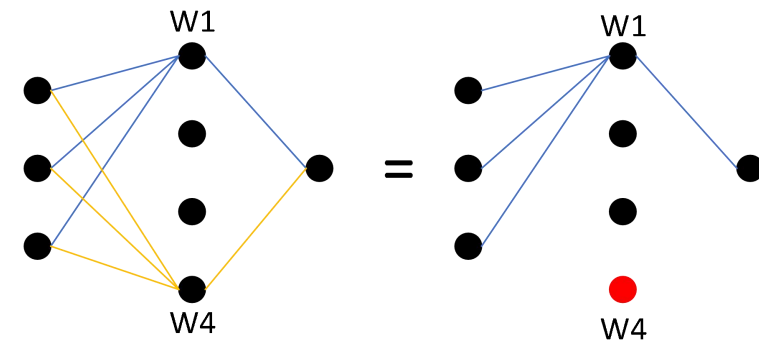

Fig. 1: A simple example showing a similar weight pair(W1=W2), we remove W4.

Deep Fried Convnets [8] is a compression method applied to the fully connected layer. It introduces a novel Adaptive Fast-food transform to reparameterize the matrix-vector multiplication of fully connected layers. The Convolutional Neural Network with Adaptive Transform, called Deep Fried Convnets, achieves the same predictive performance on ImageNet using only half the parameters of a standard network.

The third method we introduce is SVD (Singular Value Decomposition), which is usually applied to compress the fully connected network. It introduces an intermediate layer, which contains c neurons, reducing the weight matrix size from $\mathrm{m} * \mathrm{n}$ to $(\mathrm{m}+\mathrm{n}) * \mathrm{c}$. Thus, it reduces the computational and storage requirements. This method can effectively reduce the number of parameters. Denton et al. [9] used the linear characteristic of convolutional neural network based on SVD to compress AlexNet network by 5 times and control the accuracy loss within $0.9 \%$.

\section{K-means Cluster Compression}

Due to the different initial conditions in the training phase of neural networks, the trained weight does not completely converge. We can measure the intrinsic error variation in its converged state, calling the 
measured fluctuation as the confidence interval of one neural network [6]. We use these confidence intervals to determine the acceptable upper bound for the increase in prediction error due to clustering compression. For LeNet-300-100, the interval is $\pm 0.3 \%$. Table II lists the confidence intervals of four common networks.

TABLE II: THE CONFIDENCE INTERVALS OF FOUR COMMON NETWORKS

\begin{tabular}{|c|c|c|c|}
\hline & Top_1 Error & Top_5 Error & Confidence Interval \\
\hline LeNet-300-100 & $98.33 \%$ & - & $0.3 \%$ \\
LeNet-5 & $99.26 \%$ & - & $0.25 \%$ \\
Cifar10_quick & $75.75 \%$ & - & $0.34 \%$ \\
AlexNet & $58.00 \%$ & $80.86 \%$ & $0.81 \%$ \\
\hline
\end{tabular}

The idea of $K$-means clustering [10] is very classic, it is a process of moving the center of the cluster repeatedly. Firstly, we randomly select k object from n values as the initial cluster centers. Secondly, for the remaining other objects, they are respectively assigned to the nearest cluster centers. Thirdly, we update these cluster centers by calculating the mean of all objects in one cluster. Finally, we repeat above process until the value of mean remains unchanged. We apply this algorithm on the compression of neural networks. Figure 2 illustrates how it can be applied to a neural network and achieve compression.

\begin{tabular}{|c|c|c|c|c|c|c|c|c|c|c|c|}
\hline \multicolumn{4}{|c|}{ Weight(32 bit float) } & & & & & \multicolumn{4}{|c|}{ Index(2 bit) } \\
\hline 2.21 & 3.15 & 4.5 & 2.99 & & 11 & 3: & 4.50 & 1 & 2 & 3 & 3 \\
\hline 1.79 & 4.4 & -1.02 & 3.27 & Cluster\&Encoding & 10 & 2: & 3.15 & 1 & 3 & 0 & 2 \\
\hline-1.03 & 3.31 & 4.6 & 2.12 & & 01 & 1: & 2.00 & 0 & 2 & 3 & 1 \\
\hline-0.98 & 3.03 & 1.88 & -0.97 & & 00 & 0 : & -1.00 & 0 & 2 & 1 & 0 \\
\hline
\end{tabular}

Fig. 2: The process of K-means compression.

Before clustering, the weight matrix stores the 32-bit precision weight operands. After clustering and encoding the clustered values, the weight matrix stores the index of each weight. The bit-wide of the index value (determined by the number of cluster) is much smaller than the bit-wide of operands. In this way, we achieve the purpose of compression. Compression rate is calculated as:

$$
\mathrm{r}=\frac{N \times b}{N_{c} \times b+N_{f} \times \log _{2} k+k \times b}
$$

$\mathrm{r}$ represents the compression rate, $\mathrm{N}$ denotes the numbers of all weights, $\mathrm{b}$ is the bit-wide of weight, $\mathrm{k}$ represents $\mathrm{k}$ clusters, we need $\log _{2} k$ bits to encode the index. $N_{c}$ represents the weights of convolutional layer, $N_{f}$ represents the weights of full connectional layer.

\section{Experiment}

We select NVIDIA GTX 1080 and Ubuntu 16.04 as experimental platform, then choose Caffe and Keras (two ML frameworks) to quickly get the loss of accuracy after clustering compression.

\subsection{Dataset}

We select three datasets - MNIST [11], Cifar-10 [12] and ILSVRC2012 [13]. MNIST is a handwritten digit set. Cifar-10 contains $60,00032 * 32$ color images (50,000 training pictures and 10,000 test pictures). It is divided into 10 types. ILSVRC 2012 is the datasets of 2012 ILSVRC competition (ImageNet Large Scale Visual Recognition Challenge). It is the most popular large-scale classification dataset and contains 1000 categories and $100,000+$ training pictures.

\subsection{Benchmark}

We choose five representative neural networks shown in Table 1 as our benchmarks, which LeNet-300100 is a fully connected network, LeNet-5 is a classic convolution neural network, and Cifar10_quick is a simple model form open source project of Caffe. AlexNet is the champions of ILSVRC 2012. 


\subsection{Method}

We cluster every fully connected layer of the network. In order to get the best compression rate. Firstly, we choose the optimal k-value for each fully connected layer. Secondly, we select an optimal k-value combination for the whole network. Finally, we compare our method with previous compression work.

\section{Result and Analysis}

Table III-VI shows the variation of the prediction accuracy with different clusters of each fully connected layer in four neural networks. From the table, we can see that for any fully connected layer in any network, the change from 1 to 2 for $\mathrm{k}$ ( $\mathrm{k}$ denotes the number of cluster) will lead to a sharp increase in the prediction accuracy of the entire network. Then, from 2 to 8 for $\mathrm{k}$, the prediction accuracy increases slightly. When $\mathrm{k}=8$, the prediction accuracy of the network after clustering compression is very close to the uncompressed network. With k-value continues increasing, the prediction accuracy of the network begins to show small fluctuations. In this case, the fluctuations have been in confidence interval.

\begin{tabular}{|c|c|c|c|c|c|c|c|c|}
\hline & $\begin{array}{l}\text { Weight } \\
\text { rate }\end{array}$ & $\mathrm{k}=1$ & $\mathrm{k}=2$ & $\mathrm{k}=4$ & $\mathrm{k}=8$ & $\mathrm{k}=16$ & $\mathrm{k}=32$ & $\mathrm{k}=64$ \\
\hline Fc1 & $88.33 \%$ & $9.74 \%$ & $90.68 \%$ & $96.83 \%$ & $98.35 \%$ & $98.38 \%$ & $98.39 \%$ & $98.42 \%$ \\
\hline $\mathrm{Fc} 2$ & $11.30 \%$ & $9.74 \%$ & $95.95 \%$ & $98.06 \%$ & $98.36 \%$ & $98.43 \%$ & $98.45 \%$ & $98.44 \%$ \\
\hline $\mathrm{Fc} 3$ & $0.37 \%$ & $9.74 \%$ & $97.95 \%$ & $98.24 \%$ & $98.34 \%$ & $98.44 \%$ & $98.45 \%$ & $98.44 \%$ \\
\hline \multicolumn{9}{|c|}{ TABLE IV: LENET-5 (99.26\%) } \\
\hline & $\begin{array}{l}\text { Weight } \\
\text { rate }\end{array}$ & $\mathrm{k}=1$ & $\mathrm{k}=2$ & $\mathrm{k}=4$ & $\mathrm{k}=8$ & $\mathrm{k}=16$ & $\mathrm{k}=32$ & $\mathrm{k}=64$ \\
\hline Fc1 & $77.98 \%$ & $9.74 \%$ & $97.86 \%$ & $98.86 \%$ & $99.14 \%$ & $99.26 \%$ & $99.21 \%$ & $99.24 \%$ \\
\hline $\mathrm{Fc} 2$ & $16.47 \%$ & $9.74 \%$ & $99.10 \%$ & $99.17 \%$ & $99.21 \%$ & $99.29 \%$ & $99.25 \%$ & $99.26 \%$ \\
\hline $\mathrm{Fc} 3$ & $1.38 \%$ & $9.74 \%$ & $99.07 \%$ & $99.07 \%$ & $99.23 \%$ & $99.29 \%$ & $99.24 \%$ & $99.26 \%$ \\
\hline \multicolumn{9}{|c|}{ TABLE V: CIFAR10_QUICK (75.75\%) } \\
\hline & $\begin{array}{l}\text { Weight } \\
\text { rate }\end{array}$ & $\mathrm{k}=1$ & $\mathrm{k}=2$ & $\mathrm{k}=4$ & $\mathrm{k}=8$ & $\mathrm{k}=16$ & $\mathrm{k}=32$ & $\mathrm{k}=64$ \\
\hline Fc1 & $42.66 \%$ & $10.14 \%$ & $65.64 \%$ & $71.06 \%$ & $74.68 \%$ & $75.64 \%$ & $75.84 \%$ & $75.74 \%$ \\
\hline $\mathrm{Fc} 2$ & $0.42 \%$ & $10.14 \%$ & $59.88 \%$ & $72.58 \%$ & $75.80 \%$ & $75.58 \%$ & $75.74 \%$ & $75.78 \%$ \\
\hline \multicolumn{9}{|c|}{ TABLE VI: ALEXNET (TOP_1:58.00\% TOP_5:80.64\%) } \\
\hline & $\begin{array}{l}\text { Weight } \\
\text { rate }\end{array}$ & $\mathrm{k}=1$ & $\mathrm{k}=2$ & $\mathrm{k}=4$ & $\mathrm{k}=8$ & $\mathrm{k}=16$ & $\mathrm{k}=32$ & $\mathrm{k}=64$ \\
\hline Fc1 & $60.52 \%$ & $\begin{array}{c}\text { Top_1: } \\
00.12 \% \\
\text { Top_5: } \\
00.52 \%\end{array}$ & $\begin{array}{c}\text { Top_1: } \\
45.44 \% \\
\text { Top_5: } \\
71.60 \%\end{array}$ & $\begin{array}{c}\text { Top_1: } \\
57.28 \% \\
\text { Top_5: } \\
80.46 \%\end{array}$ & $\begin{array}{c}\text { Top_1: } \\
57.96 \% \\
\text { Top_5: } \\
80.36 \%\end{array}$ & $\begin{array}{c}\text { Top_1: } \\
58.04 \% \\
\text { Top_5: } \\
80.60 \%\end{array}$ & $\begin{array}{c}\text { Top_1: } \\
57.88 \% \\
\text { Top_2: } \\
80.48 \%\end{array}$ & $\begin{array}{l}\text { Top_1: } \\
57.68 \% \\
\text { Top_5: } \\
80.76 \%\end{array}$ \\
\hline Fc2 & $26.90 \%$ & $\begin{array}{c}\text { Top_1: } \\
00.12 \% \\
\text { Top_5: } \\
00.56 \%\end{array}$ & $\begin{array}{c}\text { Top_1: } \\
13.36 \% \\
\text { Top_5: } \\
27.88 \%\end{array}$ & $\begin{array}{c}\text { Top_1: } \\
57.36 \% \\
\text { Top_5: } \\
80.60 \%\end{array}$ & $\begin{array}{c}\text { Top_1: } \\
57.92 \% \\
\text { Top_1: } \\
80.60 \%\end{array}$ & $\begin{array}{l}\text { Top_1: } \\
57.84 \% \\
\text { Top_5: } \\
80.64 \%\end{array}$ & $\begin{array}{c}\text { Top_1: } \\
57.88 \% \\
\text { Top_5: } \\
80.56 \%\end{array}$ & $\begin{array}{r}\text { Top_1: } \\
58.04 \% \\
\text { Top_3: } \\
\text { 80.48\% }\end{array}$ \\
\hline $\mathrm{Fc} 3$ & $6.568 \%$ & $\begin{array}{c}\text { Top_1: } \\
00.12 \% \\
\text { Top_5: } \\
00.52 \%\end{array}$ & $\begin{array}{c}\text { Top_1: } \\
45.44 \% \\
\text { Top_1: } \\
71.60 \%\end{array}$ & $\begin{array}{c}\text { Top_1: } \\
57.28 \% \\
\text { Top_1: } \\
80.48 \%\end{array}$ & $\begin{array}{c}\text { Top_1: } \\
57.80 \% \\
\text { Top_1: } \\
80.60 \%\end{array}$ & $\begin{array}{c}\text { Top_1: } \\
57.96 \% \\
\text { Top_5: } \\
80.72 \%\end{array}$ & $\begin{array}{c}\text { Top_1: } \\
58.12 \% \\
\text { Top_5: } \\
80.56 \%\end{array}$ & $\begin{array}{c}\text { Top_1: } \\
57.92 \% \\
\text { Top_5: } \\
80.64 \%\end{array}$ \\
\hline
\end{tabular}

By analyzing the experimental results, we find that selecting appropriate value of $\mathrm{k}$ can compress the network well and let the prediction accuracy of the network not fluctuate beyond the confidence interval of the network.

Next, we firstly discuss the local optimal k-value of each fully connected layer. The case of $k<4$ is left out, because the fluctuation of prediction accuracy is not within the confidence interval in this case. When $\mathrm{k}>$ $=4$, intuitively, we think if the fully connected layer is closer to the output layer, the more clusters it should set, so that the clustering operation would have less impact on the output. However, the final experimental 
results show that when $\mathrm{k}>=4$, the prediction accuracy of the network does not increase with the increase of $\mathrm{k}$-value. Actually, it shows a trend of fluctuation. We find that the first fully connected layer may choose $\mathrm{k}=$ 32 as the optimal cluster, but the second fully connected layer may choose $\mathrm{k}=16$ as the optimal cluster. Therefore, selecting the value of $\mathrm{k}$ based on the distance from the output can only be used as a reference. In fact, we should select the value of $\mathrm{k}$ as small as possible in the premise of ensuring the prediction accuracy within the confidence interval.

We have discussed the optimal k-value of each fully connected layer. For the whole network, we find that if we opt all the local optimal k-values to cluster each fully connected layer, the final result may not be optimal. Taking AlexNet as an example, we select optimal $\mathrm{k}$ for each fully connected layer. $\mathrm{Fc} 1 \mathrm{Fc} 1$ represents the first fully connected layer) sets $\mathrm{k}=16, \mathrm{Fc} 2$ sets $\mathrm{k}=64$, and Fc3 sets $\mathrm{k}=32$. We get top_1 accuracy of $57.60 \%$ and top_5 accuracy of $80.56 \%$. This prediction accuracy is slightly lower than the top_1 accuracy of $57.84 \%$ and top_5 accuracy of $80.72 \%$. The latter is the accuracy when we set $\mathrm{k}=32$ for all fully connected layers. But the accuracy of the two schemes are within the confidence interval. When we determine the k-value of all fully connected layers in a network, we first consider using its local optimal kvalue combination. If the prediction accuracy of the clustered network is within the confidence interval, we use these k-values combination. If there is a better k-value combination, a larger compression rate can be achieved and the accuracy is not reduced (or the reduction range is within the confidence interval), then we choose the new k-value combination.

Table VII shows the k-value combinations of four networks and their prediction accuracy. Table VIII shows the compression rate and prediction accuracy of AlexNet using clustering and other compression methods. It can be seen from the two tables that the clustering compression method achieves larger compression rate and smaller accuracy reduction than other compression methods.

\begin{tabular}{|c|c|c|c|}
\multicolumn{4}{|c|}{ TABLE VII: (KI: K OF THE I-TH FULL CONNECTION LAYER) } \\
\hline & $\mathrm{k}$ & Accuracy & Compress Rate \\
\hline LeNet-300-100 & $\mathrm{k} 1=8, \mathrm{k} 2=8, \mathrm{k} 3=8$ & $98.22 \%$ & $9.12 \%$ \\
LeNet-5 & $\mathrm{k} 1=8, \mathrm{k} 2=8, \mathrm{k} 3=8$ & $99.14 \%$ & $8.98 \%$ \\
Cifar10_quick & $\mathrm{k} 1=16, \mathrm{k} 2=8$ & $75.56 \%$ & $45.23 \%$ \\
AlexNet & $\mathrm{k} 1=8, \mathrm{k} 2=8, \mathrm{k} 3=8$ & Top_1: $79.44 \%$ & $8.74 \%$ \\
& & Top_5: $80.04 \%$ & \\
\hline
\end{tabular}

\begin{tabular}{|c|c|c|c|}
\hline \multicolumn{2}{|c|}{ TABLE VIII: COMPARISON WITH OTHER COMPRESSION METHODS ON ALEXNET } \\
\hline Method & Top_1 & Top_5 & Compress Rate \\
\hline Baseline Caffemodel [14] & $58.00 \%$ & $80.64 \%$ & $1 \times$ \\
Data-free pruning [7] & $56.60 \%$ & - & $1.5 \times$ \\
Fastfood-16-AD [8] & $58.07 \%$ & - & $2 \times$ \\
Fastfood-32-AD [8] & $57.10 \%$ & - & $3.7 \times$ \\
SVD [9] & $55.98 \%$ & $79.44 \%$ & $5 \times$ \\
Kmeans Cluster Compress & $57.60 \%$ & $80.04 \%$ & $11.4 \times$ \\
\hline
\end{tabular}

\section{Conclusion}

We propose a clustering compression method. Compared with the previous compression work, it is simpler and easier to implement. To maintain the accuracy of prediction, traditional pruning and compression methods require a lot of time to retrain. However, the clustering compression method only needs to cluster the trained weights. Then we select the appropriate value of $\mathrm{k}$ to get better compression and constant prediction accuracy. We achieved 11.4x compression rate on AlexNet. By clustering compression, the compressed network model can be stored on-chip SRAM and reduce unnecessary off-chip DRAM accesses, so that large-scale deep neural networks can be applied to the limited-resources mobile platforms and embedded platforms.

Acknowledgements

\section{Acknowledgements}


Thank you for people who helped me to finish my study, especially to Sheng Ma and Yang Guo. This research is supported by the National Science Foundation of China under Grants 61672526, 61572508 and the Research Project of NUDT (ZK17-03-06).

\section{Reference}

[1] K. He, X. Zhang, S. Ren, and J. Sun, "Deep Residual Learning for Image Recognition," in IEEE CVPR, 2016.

[2] A. Y. Hannun, C. Case, J. Casper, B. C. Catanzaro, G. Diamos, E. Elsen, R. Prenger, S. Satheesh, S. Sengupta, A. Coates, and A. Y. Ng, "Deep speech: Scaling up end-to-end speech recognition," CoRR, vol. abs/1412.5567, 2014.

[3] Li Z, Wang Y, Zhi T, et al. A survey of neural network accelerators[J]. Frontiers of Computer Science, 2017, 11(5):746-761.

[4] Krizhevsky A, Sutskever I, Hinton G E. ImageNet classification with deep convolutional neural networks[C]// International Conference on Neural Information Processing Systems. Curran Associates Inc. 2012:1097-1105.

[5] Han S, Mao H, Dally W J. Deep Compression: Compressing Deep Neural Networks with Pruning, Trained Quantization and Huffman Coding[J]. Fiber, 2015, 56(4):3--7.

[6] Reagen B, Whatmough P, Adolf R, et al. Minerva: enabling low-power, highly-accurate deep neural network accelerators[C]// ACM/IEEE, International Symposium on Computer Architecture. IEEE, 2016:267-278.

[7] Srinivas S, Babu R V. Data-free parameter pruning for Deep Neural Networks[J]. Computer Science, 2015:28302838.

[8] Yang Z, Moczulski M, Denil M, et al. Deep Fried Convnets[C]// IEEE International Conference on Computer Vision. IEEE Computer Society, 2015:1476-1483.

[9] Denton E, Zaremba W, Bruna J, et al. Exploiting linear structure within convolutional networks for efficient evaluation[J]. 2014:1269-1277.

[10] Macqueen J. Some Methods for Classification and Analysis of MultiVariate Observations[C]// Proc. of, Berkeley Symposium on Mathematical Statistics and Probability. 1966:281-297.

[11] Lecun Y, Cortes C. The mnist database of handwritten digits[J]. 2010.

[12] Alex Krizhevsky and Geoffrey Hinton. Learning multiple layers of features from tiny images.2009.

[13] J. Deng, A. Berg, S. Satheesh, H. Su, A. Khosla, and L. Fei-Fei. ILSVRC-2012, 2012. URL http://www.imagenet.org/challenges/LSVRC/2012/.

[14] Yangqing Jia. BVLC caffe model zoo. http://caffe.berkeleyvision.org/model_zoo. 